\title{
Remote control system of disaster response robot with passive sub-crawlers considering falling down avoidance
}

\author{
Soichiro Suzuki ${ }^{1}$, Satoshi Hasegawa ${ }^{2}$ and Masayuki Okugawa ${ }^{3 *}$
}

\begin{abstract}
This paper describes a remote control system for a crawler-type mobile robot with passive sub-crawlers. This system has a great advantage because it utilizes an essential compliant mechanism that allows the angle of the sub-crawlers to be adapted to the shape of the road surface. Its operation is extremely simple, and it is only necessary to control the movement direction and driving speed in comparison with the case of controlling active sub-crawlers. However, a robot with passive sub-crawlers cannot recover from a situation in which it is stuck. The operator must select a traversable route for unknown rough terrain using only the information obtained from camera images and some sensor data from the robot. In this study, a remote control system for a crawler robot with passive sub-crawlers was developed based on a warning system. This system evaluates the currently selected route by calculating the stabilization for the robot when falling down in the roll and pitch directions. Experimental results obtained using a prototype crawler robot with passive sub-crawlers demonstrated the effectiveness of the proposed system.
\end{abstract}

Keywords: Crawler; Passivity; Predictive; Falling down avoidance; Remote control; Route selection; Stability margin

\section{Background}

Remote-controlled mobile robots are useful for searching around and inside buildings that have collapsed in a disaster. In a disaster area, to avoid the risk of secondary disasters, it is preferable to immediately deploy remote-controlled mobile robots instead of waiting for first responders such as firefighters. Disaster response robots should have high mobility on rough terrain. The movement mechanism of a crawler-type robot with subcrawlers has been applied to many disaster response robots in order to realize high mobility [1]. However, its use complicates robot operation because the operator must actively control each sub-crawler by estimating the attitude or state of the robot based on camera images and sensor information. Therefore, because of the multiple degrees of freedom involved, operators must be well trained to achieve high mobility by using remote control. A semi-autonomous control system for crawler robots has

\footnotetext{
*Correspondence: okugawa@aitech.ac.jp

${ }^{3}$ Aichi Institute of Technology, 1247 Yachigusa, Yakusa, Toyota, 4700369 Aichi, Japan

Full list of author information is available at the end of the article
}

been studied to realize both high mobility and simple operation $[2,3]$. This study focused on a crawler robot with passive sub-crawlers to realize simple operation with multiple degrees of freedom. Passive sub-crawlers can adapt to unknown rough terrain. We developed a crawler-type mobile robot for conducting search operations around a disaster area. Passive sub-crawlers called "Scott ${ }^{\mathrm{a}} \mathrm{I}$ " were adopted for this robot. In a crawler robot with passive sub-crawlers, it is only necessary to control the movement direction and driving speed, unlike in the case of controlling active sub-crawlers. However, posture control is impossible in passive sub-crawlers because the operator cannot actively control each sub-crawler, and a robot with passive sub-crawlers cannot recover from a situation in which it is stuck. A robot with passive sub-crawlers is extremely simple, but it is essential for the operator to possess considerable skill when selecting a route. The operator must select a traversable route for unknown rough terrain using only the information obtained from camera images and sensor data from the robot.

In this paper, we propose a warning system, not a semiautonomous control system, for a remote-controlled disaster response robot, because the operator should have

\section{Springer}

(c) 2014 Suzuki et al:- licensee Springer. This is an Open Access article distributed under the terms of the Creative Commons Attribution License (http://creativecommons.org/licenses/by/2.0), which permits unrestricted use, distribution, and reproduction in any medium, provided the original work is properly credited. 
the authority to make the final decision in order to realize flexible operation at all times. By evaluating the stability margin, a remote control system for a crawler robot with passive sub-crawlers is proposed that adopts the warning system with a dynamic threshold. The current route selected by the operator is evaluated by calculating the stabilization for the robot while falling down in the roll and pitch directions. An operator can prevent the robot from falling down based on the sound and signals from the warning system when the evaluation value for the stability margin is less than the threshold. The proposed system will be able to prompt the operator to select another route. A method of compensating for the disadvantages of a crawler robot with passive sub-crawlers will be proposed in this paper. This paper describes a method for evaluating the potential for falling down by using a predictive falling down margin time based on the normalized energy (NE) stability margin. The results of experiments demonstrated the effectiveness of the proposed system.

\section{Disaster response robot "Scott I"}

Figure 1(a) shows an overview of the "Scott I" crawlertype mobile robot. Table 1 lists the robot's specifications. The driving mechanism of the main crawler includes a drive motor and four sub-crawlers (front $\times 2$, rear $\times 2$ ). Figure 1(b) shows a cross-sectional view of the subcrawlers' joint part. Each rotary joint of the sub-crawlers can rotate freely and has passivity. The rotation of a sub-crawler's belt is transmitted from the main crawler.

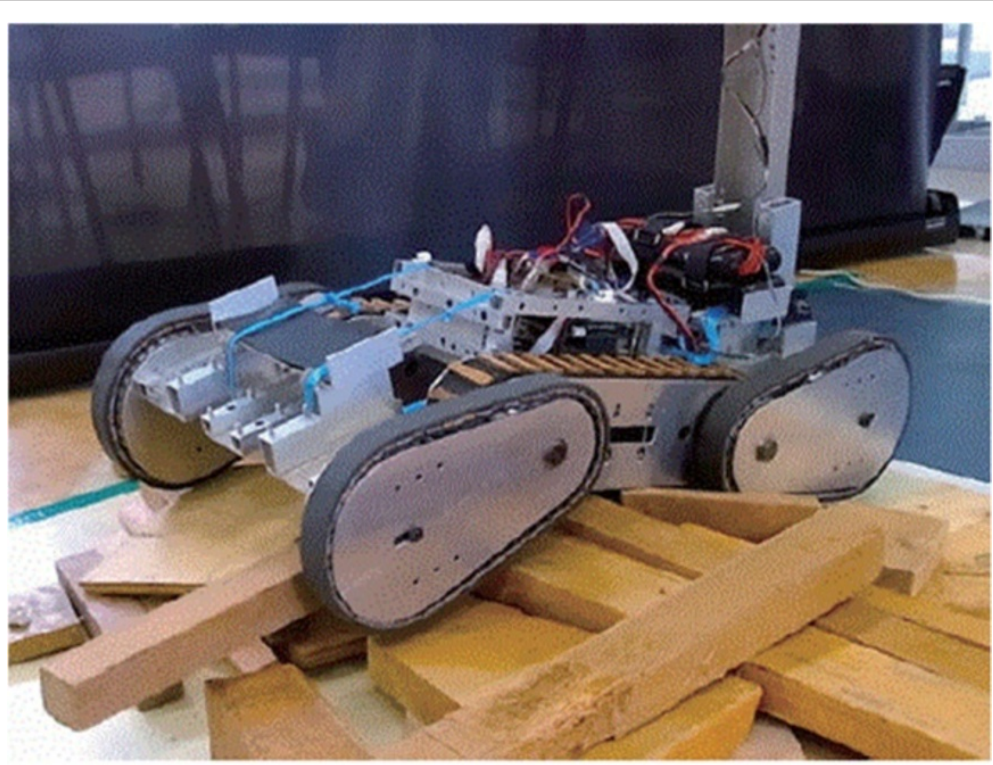

(a) Overview of "Scott I"

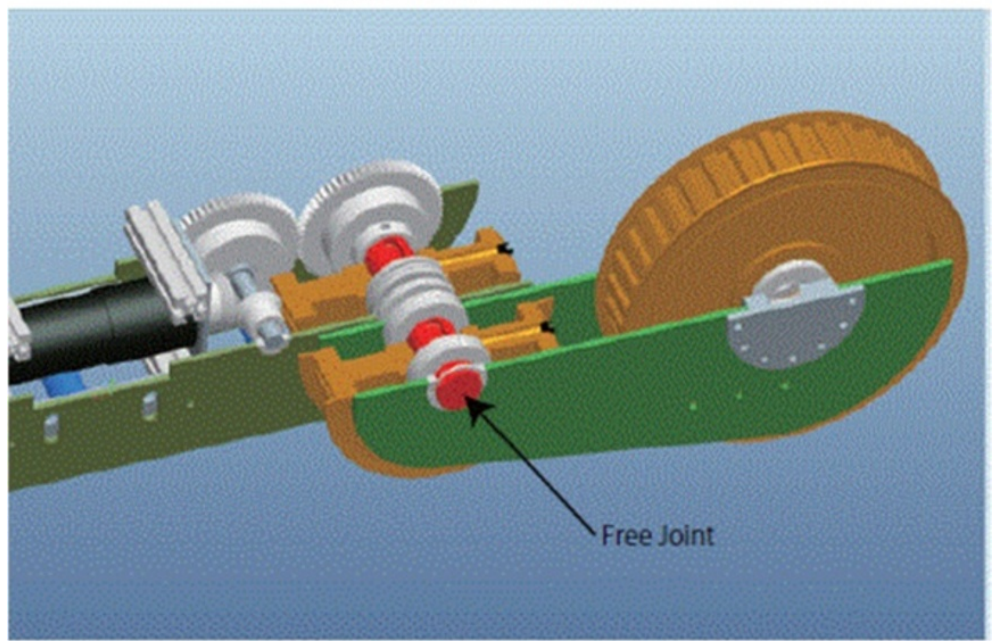

(b) Mechanism of passive sub-crawlers

Figure 1 Image of crawler-type mobile robot with passive sub-crawlers: Scott I. 
Table 1 Specifications of Scott 1

\begin{tabular}{ll}
\hline Dim. of the robot $[\mathrm{mm}]$ & Length $\times$ Width $\times$ Height \\
\hline Max. & $720 \times 390 \times 145$ \\
\hline Min. & $370 \times 290 \times 100$ \\
\hline Timing pulley dia. [mm] & 145 \\
\hline Large & 100 \\
\hline Small & \\
\hline Belt width $[\mathrm{mm}]$ & 50.8 \\
\hline Main crawler & 25.4 \\
\hline Sub-crawlers & 20.0 \\
\hline Weight $[\mathrm{kg}]$ & 0.35 \\
\hline Max. speed $[\mathrm{m} / \mathrm{s}]$ & \\
\hline
\end{tabular}

However, the rotations of the right and left sub-crawlers are not independent.

The operation of a robot with active sub-crawlers is complicated because the operator should control not only the main body but also each sub-crawler. A semiautonomous control system has been considered to overcome the disadvantage of a crawler-type robot with active sub-crawlers. We do not intend to deny the approach for a semi-autonomous control system. A crawler-type robot with passive sub-crawlers is known to have a great advantage because it has an essential compliant mechanism that allows the angle of the sub-crawlers to be adapted to the shape of the road surface $[4,5]$. The operator only needs to control the movement direction and driving speed. The effectiveness of passive sub-crawlers has been confirmed by simulations and experiments, and the mobility performance of Scott I was appreciated in RoboCup Japan Open Rescue Robot League 2013 and in the evaluation field at IRS (International Rescue System Institute in Japan). On the other hand, it is necessary to restrict the rotational angle of a sub-crawler to avoid a singular configuration when moving over a step, and numerical simulations and experiments show that the restricting angle differs with the step height and road shape. Scott I can change the restriction angle of the sub-crawlers by adjusting the length of the belt and stopper member. However, the restriction angle cannot be changed in real time. Therefore, a subject for future work will be a control method to set a suitable restriction angle based on the step height and road-surface shape.

\section{Principle of traversing on simple step for crawler-type robot with passive sub-crawlers}

The principle of traversing a simple step for a crawlertype robot with passive sub-crawlers is described. If the diameters of the pulleys for the sub-crawlers are the same, only a forward driving force is generated. Therefore, no upward power is generated, and the center of gravity of the main body of the robot cannot be moved above the obstacle. On the other hand, after the tip of a sub-crawler makes contact with an obstacle, a vertical force acts, and the sub-crawler is rotated upward by the larger pulley of the contact side. The robot will begin to traverse the step by increasing the driving force to a value greater than the weight of the robot itself.

Figure 2 shows the sequence when a crawler-type robot with passive sub-crawlers traverses a simple step. This sequence was confirmed by the results of simulations and experiments. The traversing sequence for a simple step is explained as follows. The height of a simple step is defined as greater than the radius of the large forward timing pulley, and less than the height of the center of gravity on the perpendicular robot.

1. The force acts vertically.

2. The front sub-crawlers press against the surface of the step and rotate upward.
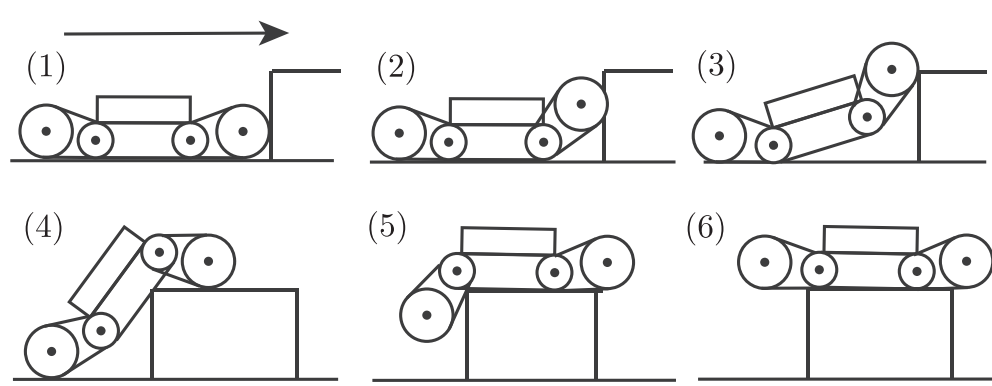

(5)

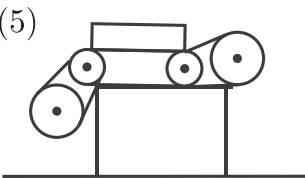

(6)
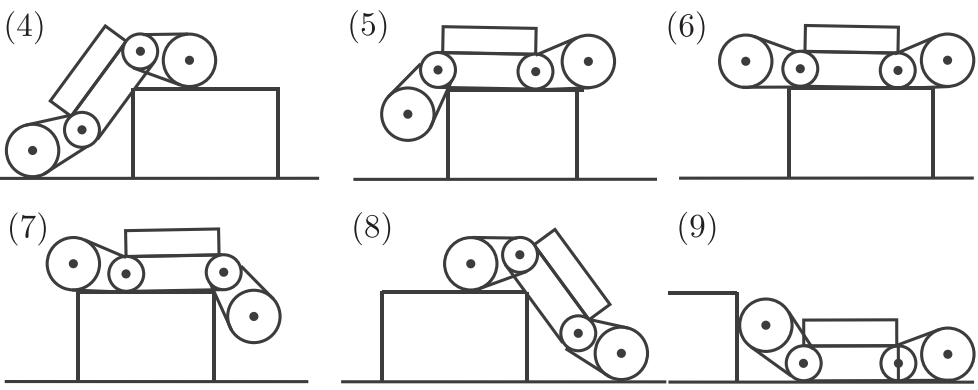

Figure 2 Sequence for traversing simple step for crawler-type mobile robot with passive sub-crawlers. 
3. The attitude angle of the main crawler increases.

4. The height of the center of gravity is higher than the objective step and moves forward from the rotational center of the rear sub-crawlers' joint.

5. The front sub-crawlers and main body move over the step.

6. The entire robot moves over the step.

7. The front sub-crawlers rotate downward.

8. The front sub-crawlers make contact with the ground.

9. The rear sub-crawlers make contact with the ground, and the robot finishes moving over the step.

\section{Warning system}

\section{Necessity of warning system}

As mentioned in the previous section, the operation is simple because a crawler robot with passive sub-crawlers can traverse rough terrain using only instructions regarding the moving direction and driving speed as long as sufficient ground pressure is applied, and the rotational angle of the sub-crawlers is appropriately restricted. However, posture control using the sub-crawlers is impossible because the operator cannot control the sub-crawlers individually. Therefore, the operator predicts the traversal of an obstacle or the state of the robot given sufficient ground pressure, and they must select an appropriate route using only the camera image and sensor information obtained from the robot (Figure 3). One problem lies in the fact that the operator must master skills for route selection in order to prevent the robot from falling down.

This paper proposes a warning system to assist the operator with route selection. This system can indicate and evaluate the stability margin for the potential of falling down by using the angle information for the robot's body in the pitch and roll directions. To prevent the robot from falling down, the proposed system can provide a warning to the operator when the stability index is below the threshold value by quantitatively evaluating the current route. A flowchart for the proposed system is shown in Figure 4. We evaluate the stability of the robot on the basis of the NE stability margin. This system has the ability to support operations in a narrow space without depending on images from an overlooking camera.

\section{Derivation of normalization energy stability margin}

The NE stability margin $E_{n}(t)$ is a stability index for a robot. It is based on the difference between the maximum height $h_{\max }$ and the current height $h(t)[\mathrm{mm}]$ for the center of gravity when the robot body rotates around the pitch and roll axes.

$$
E_{n}(t)=h_{\max }-h(t)
$$

In this study, $h_{p}(t)$ and $h_{r}(t)$ were defined as the $h(t)$ values in the pitch and the roll directions, respectively. The robot's center of gravity $h(t)$ was calculated using the body angles in the pitch $\theta_{b}(t)$ [rad], and roll $\theta_{w}(t)$ [rad] directions, along with the relative angle of the subcrawlers $\theta_{f}(t)$ to the main crawler. $\theta_{b}(t)$ was measured using a gyroscope, and $\theta_{f}(t)$ and $\theta_{r}(t)$ were measured using a potentiometer. Figure 5(a) shows the modeling of the robot and parameters for the pitch direction.

The length of the main crawler is $l_{m}[\mathrm{~mm}]$, length of the sub-crawlers is $l_{s}[\mathrm{~mm}]$, and radius of the pulley is $r[\mathrm{~mm}]$.

In the initial state, $y_{g}[\mathrm{~mm}]$ is the vertical coordinate of the center of gravity from the road surface, and $x_{g}[\mathrm{~mm}]$ is the horizontal coordinate of the center of gravity from the center of the main body. When moving up stairs, $h_{p 1}(t)$

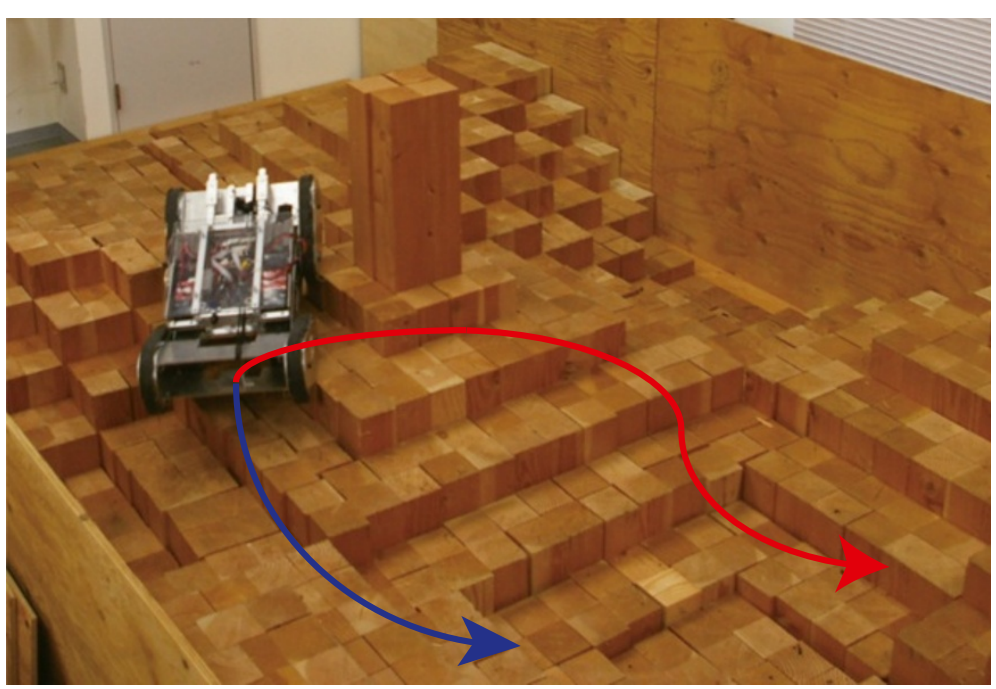

Figure 3 Which route should the operator select? 


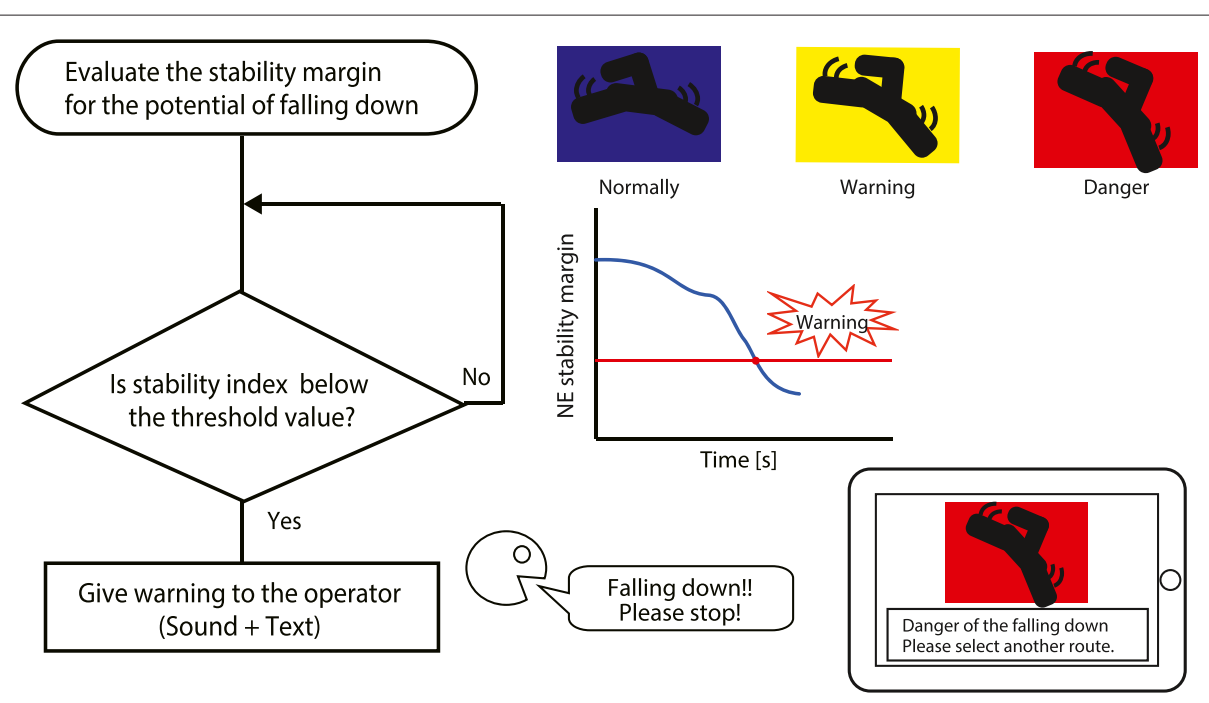

Figure 4 Flowchart for warning system.

is expressed by equation (2). When moving down stairs, $h_{p 1}^{\prime}(t)$ is expressed by equation (3).

$$
\begin{aligned}
h_{p 1}(t) & =l_{s} \sin \left(\theta_{r}(t)+\theta_{b}(t)\right)+r \\
h_{p 1}^{\prime}(t) & =l_{s} \sin \left(\theta_{f}(t)+\theta_{b}(t)\right)+r \\
h_{p 2}(t) & =\left(\frac{1}{2} l_{m}+x_{g}-y_{g} \tan \theta_{b}(t)\right) \sin \theta_{b}(t) \\
h_{p 3}(t) & =\frac{y_{g}}{\cos \theta_{b}(t)} \\
h_{p}(t) & =h_{p 1}(t)+h_{p 2}(t)+h_{p 3}(t) \\
h_{p}^{\prime}(t) & =h_{p 1}^{\prime}(t)+h_{p 2}(t)+h_{p 3}(t)
\end{aligned}
$$

The coordinate of the center of gravity for the roll direction is calculated by measuring the body angle in the roll direction $\left(-\pi / 2<\theta_{w}(t)<\pi / 2\right)$ using the gyroscope. Figure 5(b) shows the modeling of the robot and parameters in the roll direction.

$$
\begin{aligned}
h_{r 1}(t) & =\frac{1}{2} l_{w} \sin \left|\theta_{w}(t)\right|+r \cos \theta_{w}(t) \\
h_{r 2}(t) & =y_{g} \cos \theta_{w}(t) \\
h_{r}(t) & =h_{r 1}(t)+h_{r 2}(t)
\end{aligned}
$$

\section{Verification of estimated center of gravity}

The validity of equations (6), (7), and (10) is confirmed by comparing the estimated value with the experimental one. The estimated value is effective in the range of expression (11) provided that the crawler is always in contact with the ground. Where, $h_{d}$ is defined it as the height of the step.

$$
r<h_{d}<x_{g}+\frac{1}{2} l_{m}+l_{s}+r
$$

The range of the rotational angle for the sub-crawlers is limited to values between $-40 \mathrm{deg}$ and $30 \mathrm{deg}$ in order to avoid the singular configuration of the front sub-crawlers.
It is not necessary to consider the influence of the front sub-crawlers, because the rotation range is narrow. Figure 6 shows the experimental and estimated values. Figure 6(a) and (b) shows that the estimated value agrees very well with the experimental one. The error observed from 4-6 sec in Figure 6(a) occurs because the subcrawlers do not make contact with the ground. The error observed from 9-12 sec is the spike noise included in the sensor data for the angular angular velocity measured using a gyroscope when the robot moves over the step. An experiment in the roll direction was performed for a robot traversing a slope on one side of the crawler. The experimental and estimated values are shown in Figure 6(b). The roll direction of the center of gravity was exactly estimated for the sub-crawlers that were always in contact with the ground. The experimental values of the center of gravity were calculated using the coordinate obtained from image processing. The marker was attached in order to trace the center of gravity for the robot.

\section{Examination of threshold for warning system}

The timing of a warning is generated using the threshold of the NE stability margin $E_{n}(t)$. It is possible to prevent the robot from falling down if the operator takes appropriate action in response to the warning.

In this paper, a predictive falling down margin time $T_{m}$ is adopted to warn the operator that the robot will fall down. $T_{m}$ is calculated by using the time derivative for the NE stability margin $E_{n}(t)$, as shown in Figure 7. When $t=\bar{t}$, a gradient $\alpha(\bar{t})$ is defined as $d E_{n}(\bar{t}) / d t$. The equation of a tangential line for $E_{n}(t)$ at $t=\bar{t}$ is derived using equation (12).

$$
\overline{E_{n}}(t)=\alpha(t) t+E_{n}(t)
$$




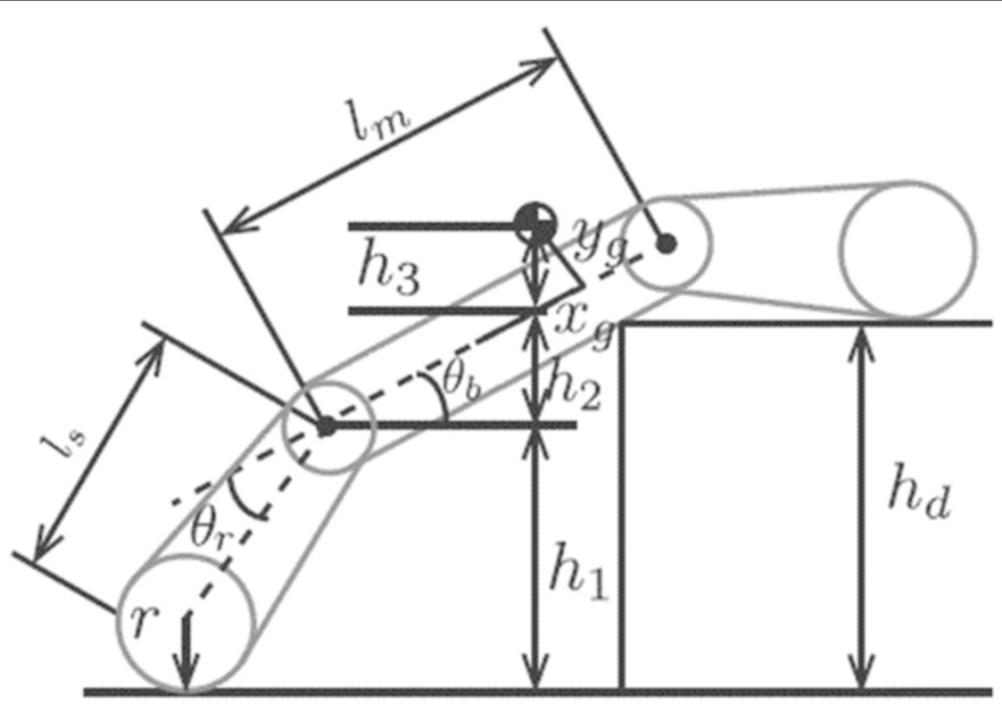

(a) Pitch direction

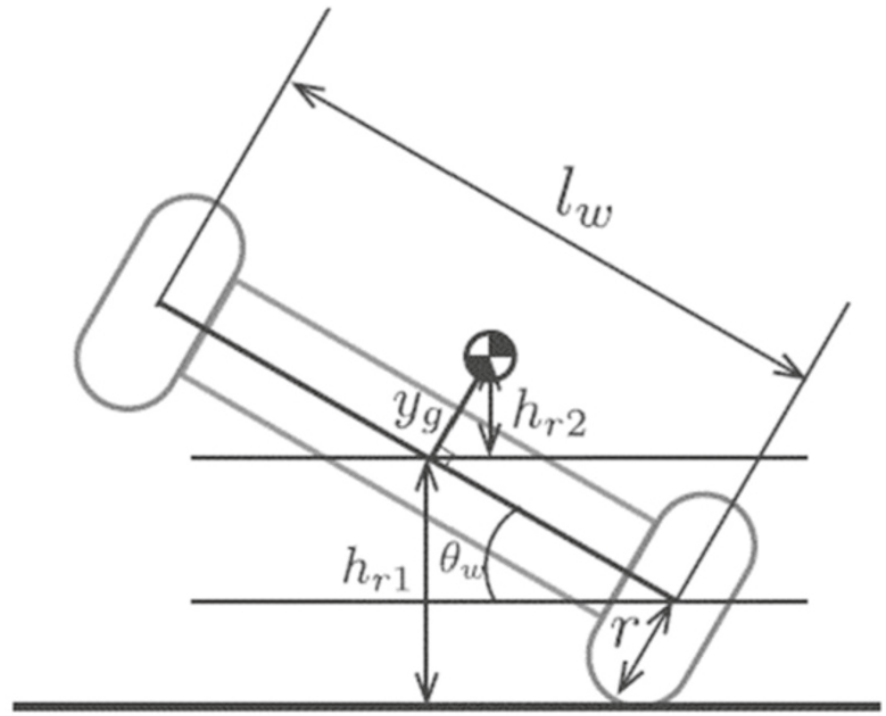

(b) Roll direction

Figure 5 Modeling of Scott I.

$T_{m}$ is defined as a time before $\overline{E_{n}}=0$ from $\bar{t}$ using equation (13).

$$
T_{m}=-\frac{E_{n}(\bar{t})}{\alpha(\bar{t})}
$$

$T_{r}$ is defined as the reaction time of the operator and $T_{e}$ is defined as the reflection time for the operator's control. The timing to give the warning should be decided in consideration of $T_{m}$ and the reaction time of the operator $T_{r}$. The reaction time is estimated as $T_{r}=0.4 \mathrm{sec}$ based on reference [6]. The reflection time is estimated to be $T_{e}=0.1 \mathrm{sec}$ in consideration of the time delay for system execution. The threshold time is determined to be $T_{m}=0.5 \mathrm{sec}$ in this paper. In addition, a notice will be displayed $0.4 \mathrm{sec}$ before the warning. The attention time is assigned while $0.5<T_{m}<1.0$.

\section{Method for operation support}

As shown in the Figure 8, the robot body posture for the pitch and roll directions and the stability will be displayed on the operation screen to determine the appropriateness of the route selection. The stability of the operation is displayed on the monitor in terms of the rate of $E_{n}(t)$ for the initial posture of the robot and the present one. Depending on the deterioration of the stability margin, the background color of the display is sequentially changed from blue, to yellow, and finally to red. If the NE stability margin is lower than the threshold, the level of danger will 


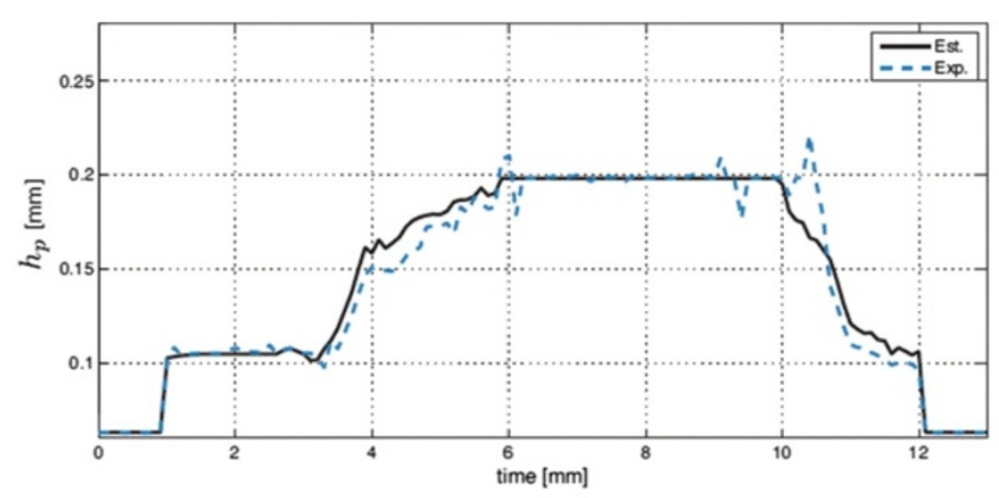

(a) Pitch direction

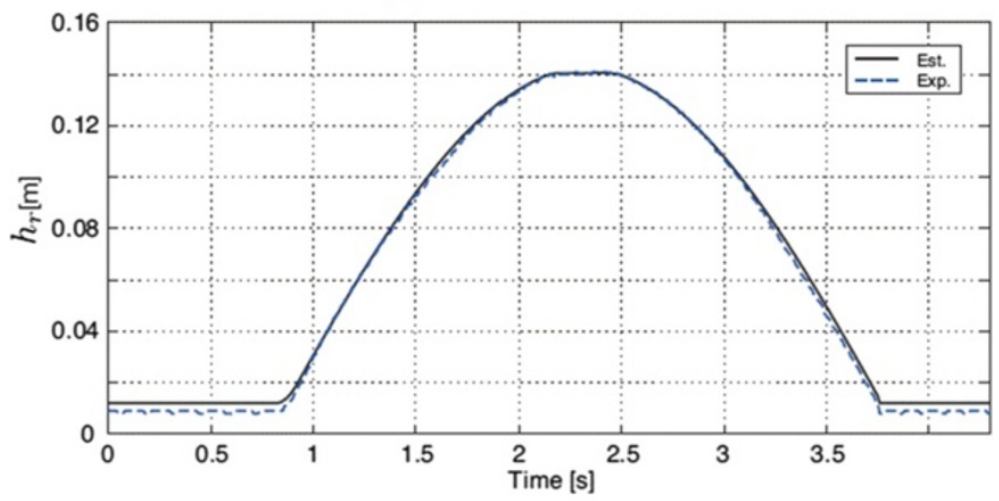

(b) Roll direction

Figure 6 Comparison of estimated and experimental values.

be presented as a warning by voice and a signal because of the risk of the robot falling down.

In the case of a robot with active sub-crawlers, the operator must appropriately control each sub-crawler's angle in every sequence. A robot may fall down if the operator lacks adequate skill even if it is a traversable step. In addition, the difference between the traveling sequences of active and passive sub-crawlers lies in the manner in which the rear sub-crawlers are utilized. Passive subcrawlers can only rotate using gravity; consequently, no

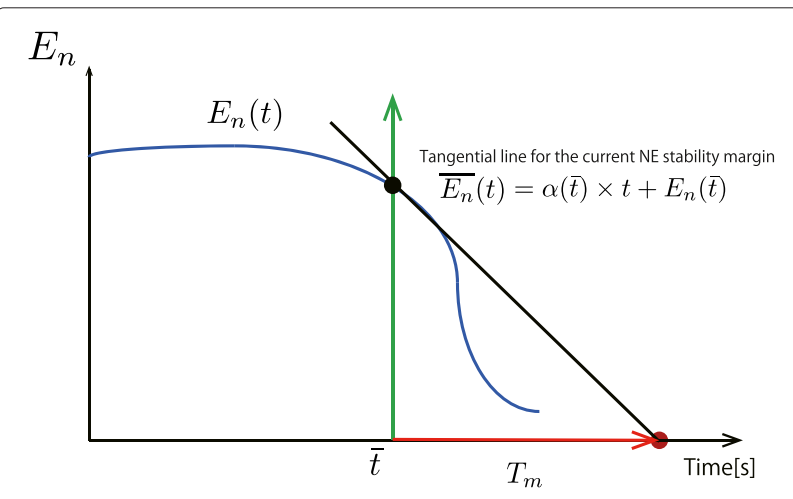

Figure 7 Image of predictive falling down margin time. downward power to lift the robot body is generated. Adopting passive sub-crawlers has many advantages. It can reduce the number of high-torque motors, reduce the robot weight, and save energy. The relationship between the traversing condition and the sub-crawlers' restricting angle is reported in another paper.

\section{Methods}

\section{Experimental operation verification using Scott I}

We confirmed the process of the proposed system using an experimental verification under a real environment. In the experiment, we observed the progress of the operator response to the warning provided by the proposed system. In the experimental condition for the pitch direction, the target obstacle was a simple step (height: $0.4 \mathrm{~m}$, depth: $0.3 \mathrm{~m}$, width: $0.8 \mathrm{~m}$ ) with an impossible height that was to be traversed. In the experimental condition for the roll direction, the target obstacle was a slope with a $30 \mathrm{deg}$ angle of inclination. The robot traversed this slope only with the left side of the crawler, as shown in Figure 9(b). The operator screen and the posture of Scott I during this experiment are shown in Figure 9.

The time history of the NE stability margin $E_{n}(t)$ and predictive falling down margin time $T_{m}(t)$ are shown in 


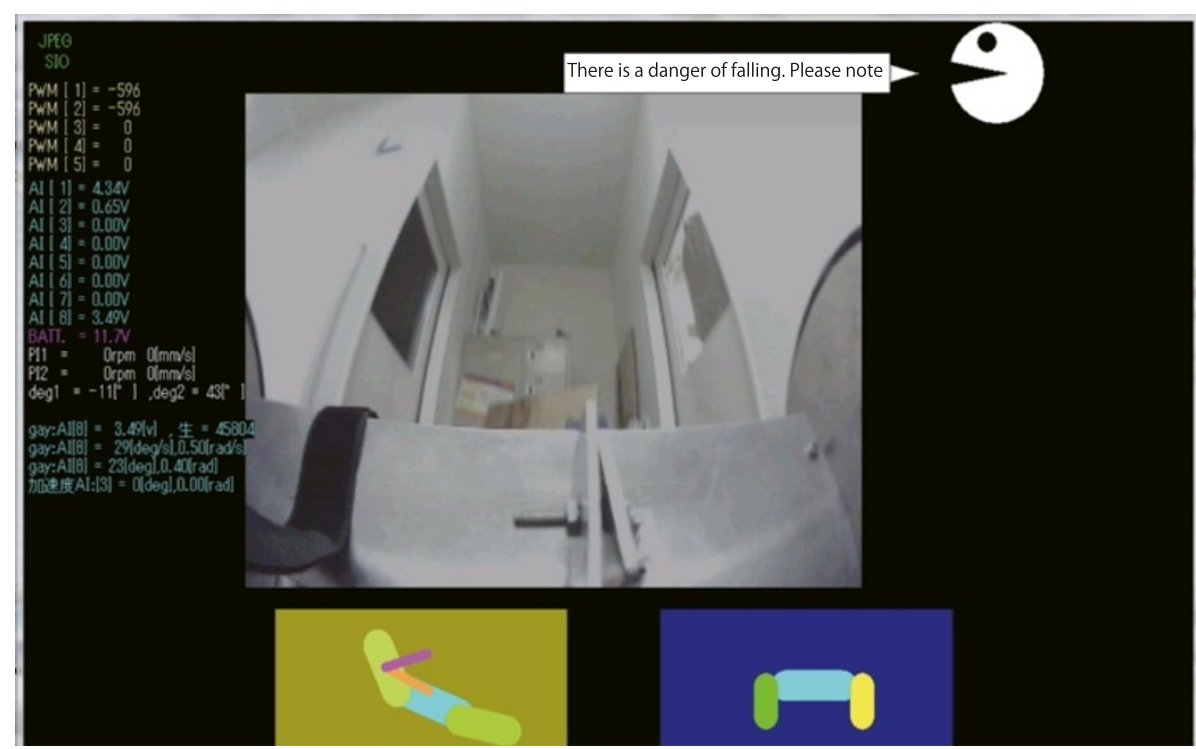

Figure 8 Display method for operation support information.

Figure 10. The experimental results demonstrate the effectiveness of the proposed system, as shown in Figure 10. We confirmed that the operator could react appropriately to prevent the robot from falling down within approximately $5.0 \mathrm{sec}$ of the warning. However, the robot fell down when the operator did not react at the warning because a strict threshold was assumed. In addition, we could not confirm the effectiveness of the attention time because of the short setting time. We would like to consider these in our future work.

\section{Experimental evaluation and consideration}

The experimentation for the evaluation of the proposed system was performed with 10 test subjects. We chose

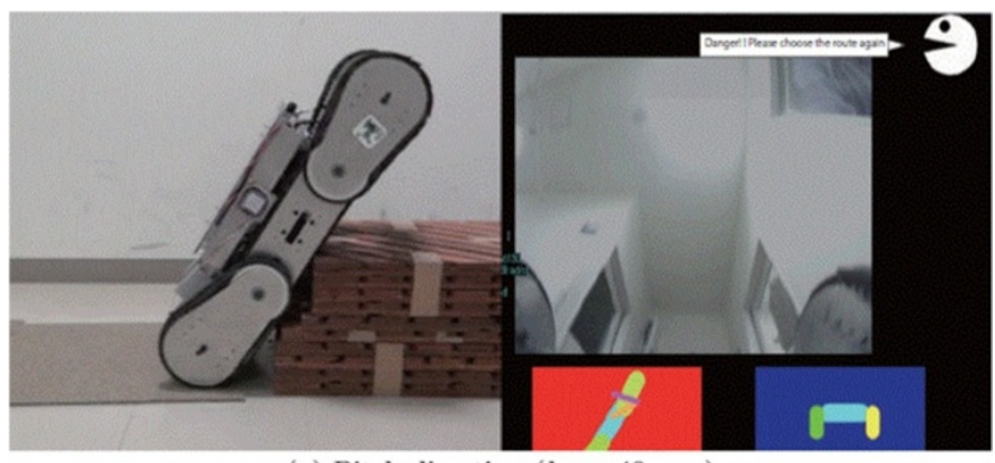

(a) Pitch direction $\left(h_{d}=40 \mathrm{~mm}\right)$

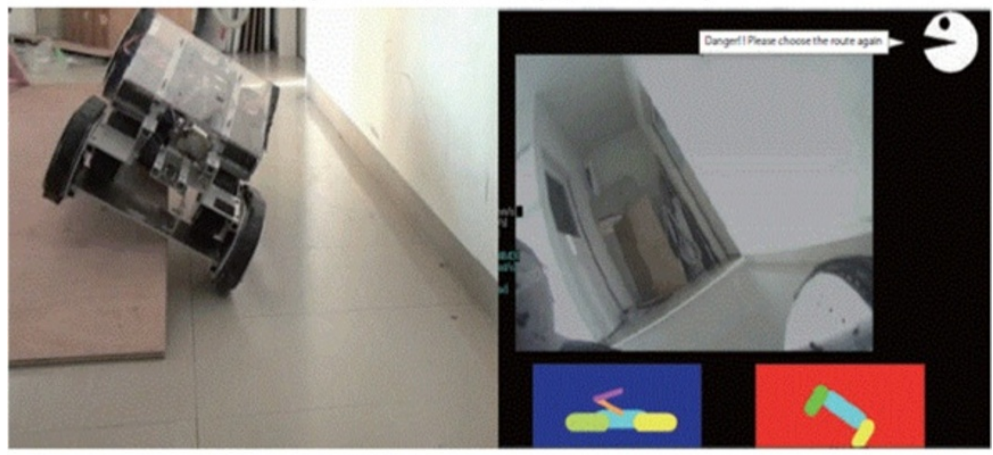

(b) Roll direction (30 deg)

Figure 9 Experimental scene and operator screen. 


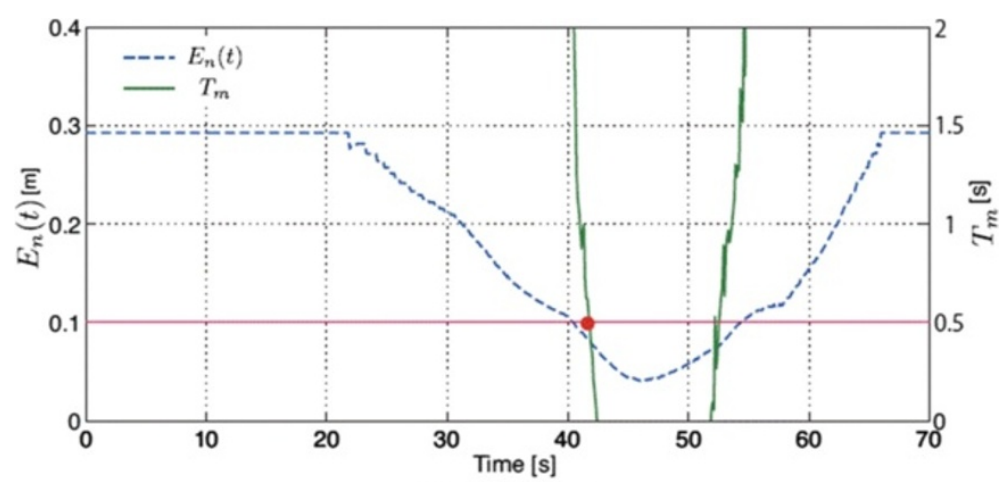

(a) Pitch direction

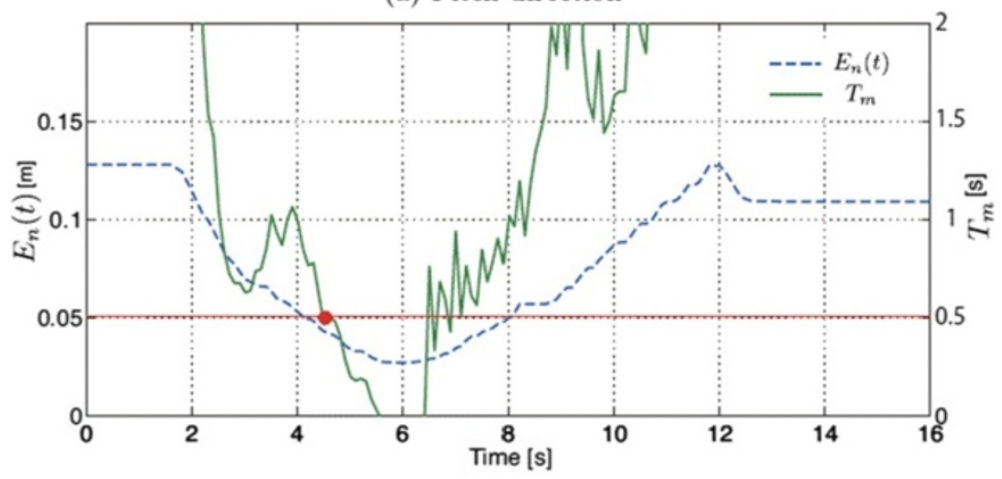

(b) Roll direction

Figure 10 Experimental operation verification of proposed system.

expert operators for half the subjects and beginner operators for the rest. On the other hand, the beginner operators were defined as inexperienced operators. The objective of the experiment was to evaluate the reaction of each subject in the operating process for traversing a simple step or slope. The experiment was performed using three cases:

Case 1 Using only the images from the front camera (shown in Figure 11(a))

Case 2 Using only the images from the overlooking camera mounted on the manipulator (shown in Figure 11(b))

Case 3 Using the proposed system.

Each subject performed the operation nine times for these experimental cases.

Equation (14) defines the height of the objective traversing step $\bar{h}$, where $h_{e}[\mathrm{~m}]$ is the maximum height of the step that the robot can traverse and $\varepsilon_{h}$ is the adjustable height of the step. The step height was classified three conditions $(\bar{h}=180,230,280 \mathrm{~mm})$, and we chose randomly it for each test subjects. Each step height condition was chosen to become the same number of experiments.

$$
\bar{h}=h_{e}+\varepsilon_{h}
$$

In the case where the step to be traversed had an untraversable height $\left(\bar{h}>h_{e}\right)$ we defined a misjudgment as the situation in which the robot fell down even though the subject could traverse the objective step and the robot could continue to move forward. We also defined a misjudgment as the situation in which the subject operated the robot backward upon understanding that the step to be traversed had an untraversable height.

The experimental results were evaluated by the misjudgment rate $R_{w}$ based on equation (15), where $E_{x}$ is the number of times the experiment was conducted and $M_{j}$ is the number of misjudgments.

$$
R_{w}=\frac{M_{j}}{E_{x}}
$$

However, the same number of experiments was performed for each step height condition. The experiments were only performed in the pitch direction at a controllable constant speed $(350 \mathrm{~mm} / \mathrm{sec})$ for a sampling period of $0.1 \mathrm{sec}$.

Figure 12 shows the experimental results for the misjudgment rate. The results for the beginner and expert operators are shown in Figure 12(a) and (b), respectively. The graphs show the misjudgment rates on the horizontal axes, and the step height conditions on a vertical axes. The values of the standard deviations for every 


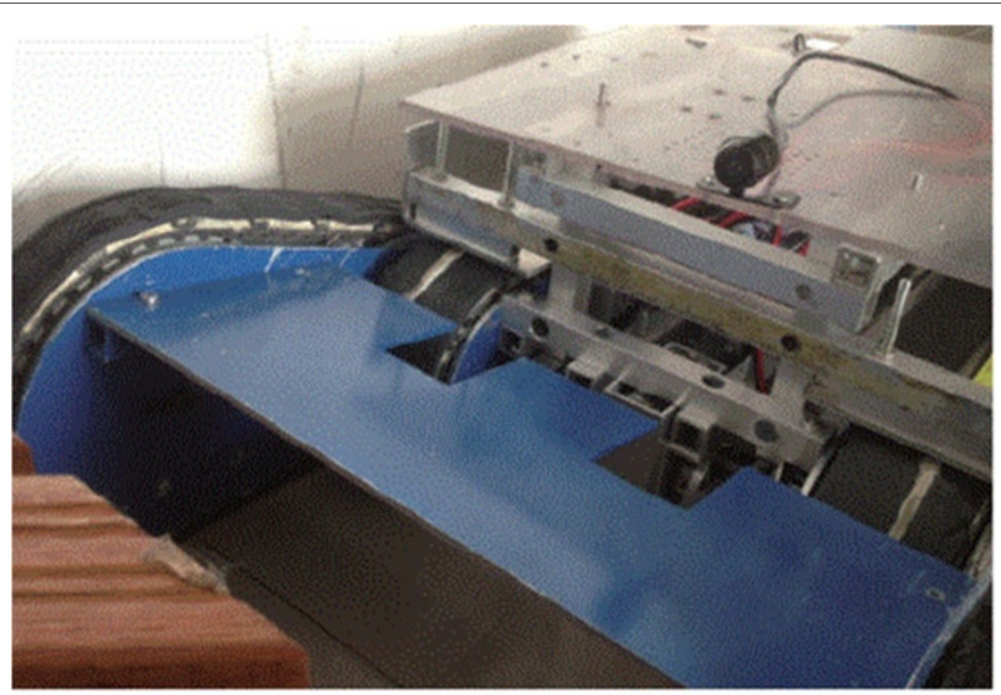

(a) Front camera mounted on main body

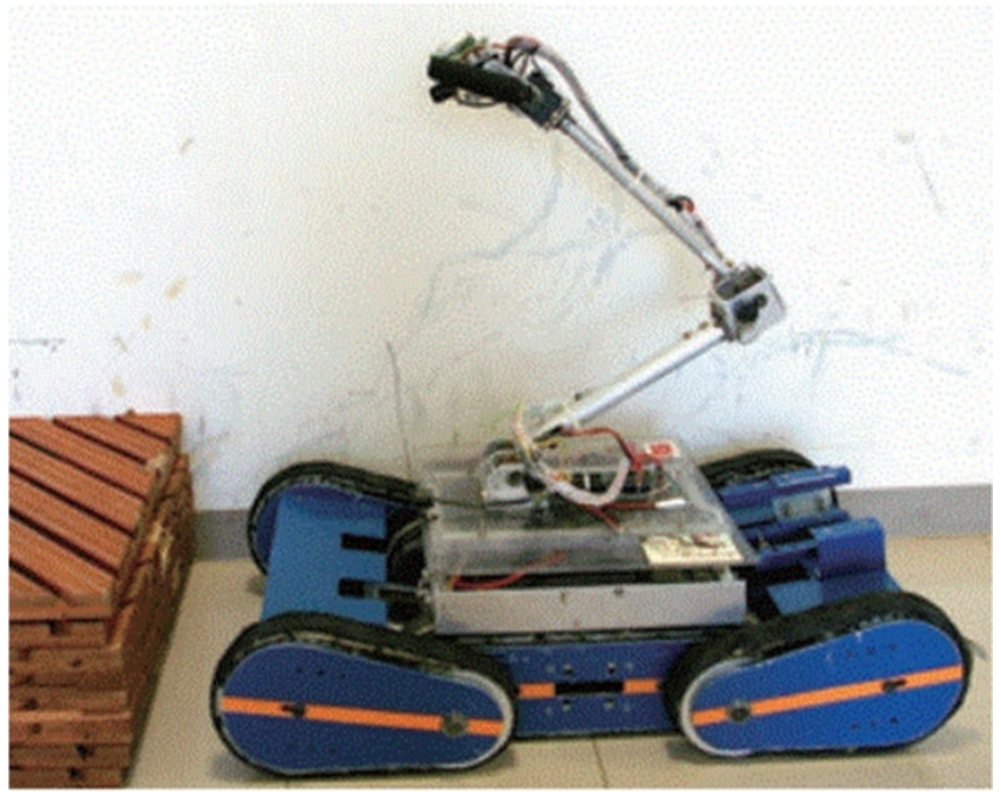

(b) Overlooking camera mounted on manipulator

Figure 11 Image of the cameras mounted on Scott.

each step height show in Table 2. The results and discussion are given below based on observing the performances of the operators and discussing these after the experimentation.

\section{Results and discussion}

First, the results for the beginners are shown in Figure 12(a). In the case of $\bar{h}>h_{e}$, the appropriate judgment is "move backward" because of the untraversable step height. When using only the images from the front camera (Case 1) or those from the overlooking camera (Case 2), the value of $R_{w}$ was high. From these results, we confirmed that when only using camera images, it is difficult for operators to respond appropriately to prevent the robot from falling down. The obstacle moves out of the camera frame whenever a robot traverses the step because the attitude angle of the robot increases. Therefore, the judgment depends on the experience of the operator. In the case of $\bar{h}>h_{e}$, we frequently observed the tendency of the cautious judgment to "move backward". As a result, the misjudgment rate in the case of $\bar{h}>h_{e}$ was smaller than the case of $\bar{h}=h_{e}$.

On the other hand, when using the warning system (Case 3), the value of $R_{w}$ was the smallest for the 


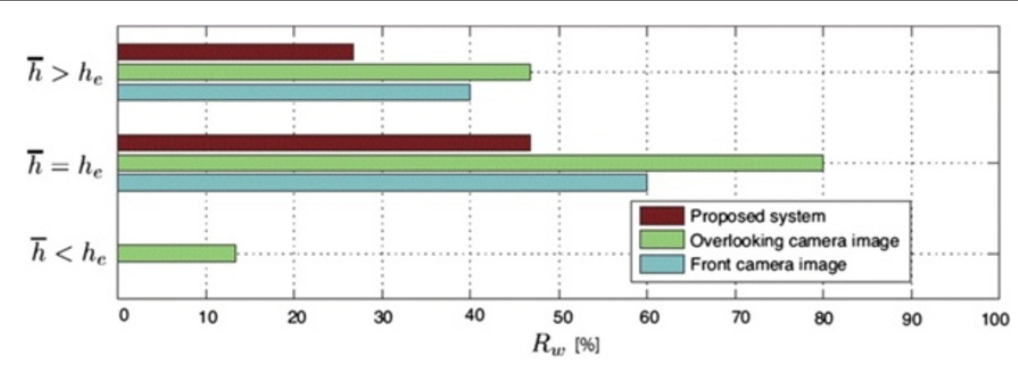

(a) Beginner operators

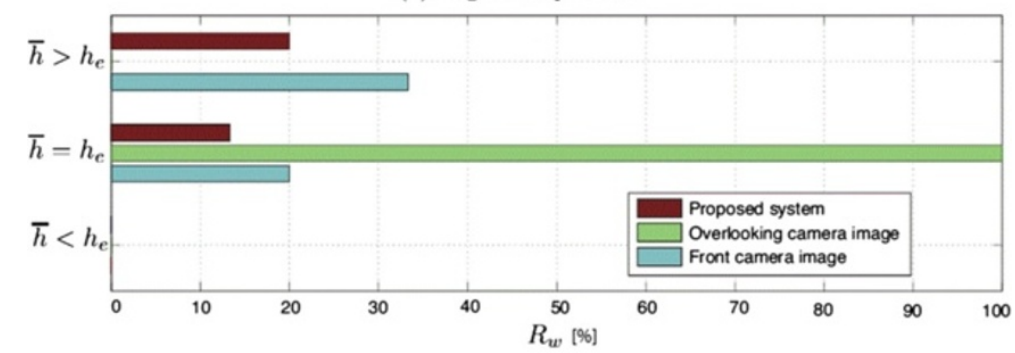

(b) Expert operators

Figure 12 Experimental results for misjudgment rate.

untraversable step height $\bar{h}>h_{e}$. The operators could appropriately control the robot to prevent it from falling down.

Second, in the case of $\bar{h}=h_{e}$, the value of $R_{w}$ was high under all of the experimental conditions. The "move backward" choice was considered a misjudgment because of the traversable step height. The reasons for Case 1 and Case 2 were similar in the case of $\bar{h}>h_{e}$. Hence, rash operations and falling down were frequently observed. The reasons for Case 3 was different. The judgment of almost all of the subjects was to "move backward," particularly when using the proposed system. By adopting the proposed warning system, the cautious judgments increased, but rash judgments decreased. The proposed system attached importance to the capability to avoid falling down. When observing the experiments for $\bar{h}=h_{e}$, it seemed that some subjects were confused because the driving speed of the robot had to be controlled in the case

Table 2 Standard deviations for misjudgment rate

\begin{tabular}{llcc}
\hline \multicolumn{2}{c}{ Step height } & Beginner & Expert \\
\hline \multirow{4}{*}{$\bar{h}>h_{e}$} & Case 1 & 38.9 & 29.8 \\
\cline { 2 - 4 } & Case 2 & 26.6 & 0 \\
\cline { 2 - 4 } & Case 3 & 24.9 & 26.7 \\
\hline \multirow{3}{*}{$\bar{h}=h_{e}$} & Case 1 & 24.9 & 26.7 \\
\cline { 2 - 4 } & Case 2 & 26.6 & 0 \\
\hline \multirow{3}{*}{$\bar{h}<h_{e}$} & Case 3 & 45.2 & 26.7 \\
\hline & Case 1 & 0 & 0 \\
\cline { 2 - 4 } & Case 2 & 26.7 & 0 \\
\cline { 2 - 4 } & Case 3 & 0 &
\end{tabular}

of the limit of the traversable step $\bar{h}=h_{e}$. This was confirmed by the standard deviation results $\left(\bar{h}=h_{e}\right.$, Case 3 , Beginner) as listed in Table 2 . The driving speed will need be considered in future work.

Finally, for the traversable step height $\bar{h} \leq h_{e}$, because the operators only had to select the simple command "Forward" in all cases, the robot was able to traverse the step. The misjudgments of the subjects were almost always "move backward" when using only images from the overlooking camera (Case 2). This result shows that increasing the information to the operator will result in more cautious judgments.

Figure 12(b) shows the experimental results for the misjudgment rate of the expert operators. This rate was smaller than that for the beginners overall. Furthermore, the value of the standard deviation is also small in the case of an expert. We confirmed that the expert operators could perform an appropriate judgments. In the case of $\bar{h}>h_{e}$, when using only a images from the overlooking camera (Case 2), all of the subjects avoided falling down. The subjects chose the more cautious judgment based on the experience gained in the previous experiments because Case 2 was the last to be performed. In the case of the warning system, we observed that a relatively inexperienced operator let the robot fall down.

In the case of $\bar{h}=h_{e}$, all of the subjects made wrong judgments for Case 2 . The reason was similar to that for the beginner operators. Hence, for Case 1, the misjudgment rate for the expert operators was smaller than the case of $\bar{h}>h_{e}$ because the obstacle did not frequently move out of the camera frame. When using the proposed system, because it was possible to adjust the 
movement speed, we confirmed that the high success rate was achieved by repeated stops and starts.

\section{Conclusion}

This paper describes a warning system for a crawler robot with passive sub-crawlers. The proposed support system for the operator could display an evaluation index for the selected route based on the NE stability margin of the robot. Experiments were performed by using Scott I to verify the proposed system. We compared the misjudgment rates between the beginner and expert operators under different experimental conditions. The experimental results demonstrated the effectiveness of the proposed system in all cases. We confirmed that the proposed system was able to stimulate a cautious judgment. Based on the experimental results, we found that it was necessary to provide suitable support based on the skill of the operator. For expert operators, it would be best for the system to provide only assistance. On the other hand, because of their lack of skill, beginner operators cannot react quickly enough to prevent the robot from falling down. Hence, in order to support a beginner operator, we think that it would be best for the system to interrupt their actions, such as through a speed-controlled system. In future work, an experiment will be performed in a real environment by using an improved system to evaluate the effectiveness of the proposed method for expert or beginner operators.

\section{Endnote}

${ }^{a}$ Abbreviation for "Scouting Crawler Robot Technology”.

Competing interests

The authors declare that they have no competing interests.

\section{Authors' contributions}

SH devised the basic concept of the overall system, technically constructed the system and drafted the manuscript. SS carried out the experiments and helped to draft the manuscript. MO suggested the constitution of the article and helped with the editing of the manuscript. All authors read and approved the final manuscript.

\section{Acknowledgements}

The authors would like to thank Sanritz Automation Co. Ltd. for the development of the computer, "TPIP". This work was supported by a JSPS KAKENHI Grant-in-Aid for Scientific Research (B), No. 23310116.

\section{Author details}

${ }^{1}$ Graduate School of Engineering, Aichi Institute of Technology, 1247 Yachigusa, Yakusa, Toyota, 4700369 Aichi, Japan. ${ }^{2}$ Daiichi System Engineering, 4F Green Bldg, 1-16-20 Nishiki Naka Nagoya, 460003 Aichi, Japan. ${ }^{3}$ Aichi Institute of Technology, 1247 Yachigusa, Yakusa, Toyota, 4700369 Aichi, Japan.

Received: 15 January 2014 Accepted: 23 May 2014

Published online: 30 November 2014

\section{References}

1. Koyanagi E (2010) Rescue robot with active sub crawlers. J Robot Soc Japan 28(2):147-150
2. Ohno K, Morimura S, Tadokoro S, Koyanagi E, Yoshida T (2007) Semiautonomous control system of rescue crawler robot having flippers for getting over unknown-steps. In: Proceedings of the IEEE/RSJ international conference on intelligent robots and systems 2007. pp 3012-3018

3. Okada Y, Nagatani K, Yoshida K, Yoshida T, Koyanagi E (2010) Shared autonomy system for tracked vehicles to traverse rough terrain based on continuous three-dimensional terrain scanning. In: Proceedings of the IEEE/RSJ international conference on intelligent robots and systems 2010 pp 357-362

4. Yuuki K, Ikarashi T, Oogane K (2010) Validity of passivity mechanism with rescue robots. In: Proceedings of annual conference of robotics society of Japan 2010. pp 3-14

5. Kang S, Lee W, Kim M, Shin K (2005) Robhaz-rescue: Rough-terrain negotiable teleoperated mobile robot for rescue mission. In: Proceedings of the IEEE/RSJ international conference on intelligent robots and systems 2010. pp 105-110

6. Teichner WH, Krebs MJ (1974) Laws of visual choice reaction time. Psycho Rev 81(1):75-98

doi:10.1186/s40648-014-0020-9

Cite this article as: Suzuki et al:: Remote control system of disaster response robot with passive sub-crawlers considering falling down avoidance. ROBOMECH Journal 2014 1:20

\section{Submit your manuscript to a SpringerOpen ${ }^{\mathcal{O}}$ journal and benefit from:}

- Convenient online submission

- Rigorous peer review

- Immediate publication on acceptance

- Open access: articles freely available online

- High visibility within the field

- Retaining the copyright to your article

Submit your next manuscript at $>$ springeropen.com 